\title{
Context-dependent differences in miR-10b breast oncogenesis can be targeted for the prevention and arrest of lymph node metastasis
}

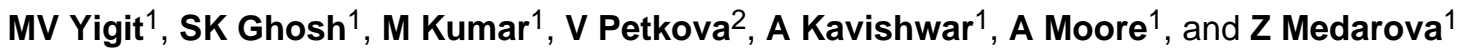 \\ ${ }^{1}$ Department of Radiology, Molecular Imaging Laboratory, Athinoula A Martinos Center for \\ Biomedical Imaging, Massachusetts General Hospital and Harvard Medical School, Charlestown, \\ MA, USA \\ ${ }^{2}$ Beth Israel Deaconess Medical Center, Harvard Medical School, Boston, MA, USA
}

\section{Abstract}

\begin{abstract}
Metastases, and not the primary tumor from which they originate, are the main reason for mortality from carcinoma. Although the molecular mechanisms behind metastasis are poorly understood, it is clear that epigenetic dysregulation at the level of microRNA expression is a key characteristic of the metastatic process that can be exploited for therapy. Here, we describe an miRNA-targeted therapeutic approach for the prevention and arrest of lymph node metastasis. Therapy relies on the inhibition of the pro-metastatic microRNA-10b. It is delivered to primary and lymph node metastatic tumor cells using an imaging-capable nanodrug that is designed to specifically home to these tissues. Treatment of invasive human breast tumor cells (MDAMB-231) with the nanodrug in vitro downregulates miR-10b and abolishes the invasion and migration of the tumor cells. After intravenous delivery to mice bearing orthotopic MDAMB-231-luc-D3H2LN tumors, the nanodrug accumulates in the primary tumor and lymph nodes. When treatment is initiated before metastasis to lymph nodes, metastasis is prevented. Treatment after the formation of lymph node metastases arrests the metastatic process without a concomitant effect on primary tumor growth raising the possibility of a context-dependent variation in miR-10b breast oncogenesis.
\end{abstract}

\author{
Keywords \\ imaging; miRNA; metastasis; miR-10b; nanoparticle; therapy
}

\section{INTRODUCTION}

The process of metastasis occurs in two major phases: (i) physical translocation of cancer cells from the primary tumor to a distant organ and (ii) colonization of that organ by tumor cells. Invariably, the first step in the metastatic cascade is the acquisition of an invasive phenotype by the tumor cells that permits local colonization of the surrounding tissue and entry into the microvasculature of the lymph and/or blood systems. ${ }^{1}$

\footnotetext{
(C) 2013 Macmillan Publishers Limited All rights reserved

Correspondence: Dr A Moore or Dr Z Medarova, Department of Radiology, Molecular Imaging Laboratory, MGH/MIT/HMS Athinoula A Martinos Center for Biomedical Imaging, Massachusetts General Hospital/Harvard Medical School, Building 75, 13th Street, Charlestown, MA 02129, USA. amoore@ @elix.mgh.harvard.edu or zmedarova@ partners.org.

CONFLICT OF INTEREST The authors declare no conflict of interest.

Supplementary Information accompanies the paper on the Oncogene website (http://www.nature.com/onc)
} 
Recently, several microRNAs have been identified that mediate the process of tumor cell migration and tissue invasion. ${ }^{2-6}$ One of these microRNAs, miR-10b, is involved in the metastasis of a number of adenocarcinomas, including breast, ${ }^{2,6}$ lung, colon and bladder cancers. $^{7}$

In the present study, we focused on targeting miR-10b, with the goal of alleviating breast cancer metastasis. We reasoned that using an miR-10b-inhibiting nanodrug (MN-antimiR10b) capable of homing to primary and lymph node metastatic tumor cells, we would be able to significantly decrease the ability of primary tumor cells to metastasize to locoregional lymph nodes.

The physicochemical properties of the synthesized nanodrug promote mechanical targeting to the tissues of interest, while the molecular design facilitates specific delivery to the tumor cells within these tissues. In particular, the design of the nanodrug (MN-anti-miR10b) features ultrasmall magnetic nanoparticles $(\mathrm{MN})^{8,9}$ with RGD (tripeptide arginine-glycineaspartic acid)-targeting capabilities ${ }^{10-12}$ decorated with locked nucleic acid (LNA) oligonucleotides as a therapeutic moiety. ${ }^{13-15}$

The inherent capacity of MN-anti-miR10b for noninvasive imaging allowed for obtaining semi-quantitative information about nanodrug bioavailability in target tissues by magnetic resonance imaging (MRI) and the whole-body distribution by near infrared fluorescence optical imaging. This information could be invaluable in a clinical setting for determining the need for re-treatment on a patient-by-patient basis, opening up the possibility for designing individualized therapeutic regimens.

The size and composition of the MN used in this study promote their extended persistence in the circulation (blood half-life $>12 \mathrm{~h}$ ) and transcytosis into the tumoral and lymph node interstitium, thereby enabling a mechanical targeting of therapy to the tumoral and lymph node compartments. ${ }^{16}$ As a result of miR-10b inhibition with the nanodrug, we observed virtually complete prevention of metastatic dissemination from the primary tumor and growth arrest of already formed lymph node metastases in an animal model of breast cancer. This suggested a context-dependent role of miR-10b in metastasis. Whereas in the primary tumor, miR-10b appeared to control metastasis but not tumor growth, in the lymph node metastases, it influenced macroscopic tumor formation. We believe that the approach described here is highly relevant not only to metastatic breast cancer but also to a number of adenocarcinomas, in which miR-10b plays a role. ${ }^{17}$ On a broader scale, the delivery strategy illustrated by our studies could provide a useful research tool for the elucidation of contextdependent differences in miRNA function and the translation of this knowledge to a clinical setting.

\section{RESULTS}

\section{$M N-a n t i-m i R 10 b$ inhibits human miR-10b and reduces the invasion and migration potential of MDA-MB-231 human breast adenocarcinoma cells}

To develop an miR-10b-inhibiting nanodrug detectable by noninvasive imaging, we employed a strategy previously described by us ${ }^{8,9}$ to synthesize small amine terminated dextran-coated MN. These nanoparticles were labeled with the near infrared fluorescent dye Cy5.5 and conjugated to the tumor-targeting peptide cRGD that has a high affinity for av $\beta 3$ integrin. We functionalized the nanoparticles with LNA antisense oligonucleotides targeting human miR-10b (anti-miR-10b), known to promote tumor cell metastasis (MN-antimiR10b; Figure 1a). ${ }^{2}$ 
The nanodrug was taken up by nearly $100 \%$ of the cells, as revealed by flow cytometry (Figure 1b). In addition, all of its components were present intracellularly, as shown by confocal microscopy (Figure 1c). The nanodrug mediated an $87.8 \pm 6.2 \%$ inhibition of miR-10b (Figure 1d), indicating that our delivery method is highly efficient.

Nanodrug uptake was also studied in the highly metastatic breast cancer cell line 4T1 to demonstrate a broader application of the design. The uptake was confirmed by flow cytometry. The nanodrug mediated a significant downregulation of miR-10b levels in this cell line, which expresses very high levels of miR-10b, attesting to the robustness of our delivery approach (Supplementary Figure 1).

To study the phenotypic effects of miR-10b knockdown by the nanodrug, we evaluated the invasion and migration potential of MDA-MB-231-GFP cells treated with MN-anti-miR10b. Remarkably, MN-anti-miR10b treatment abrogated the migration (Figure 2a) and invasion (Figure 2b) capabilities of the cells.

\section{In-vivo imaging can provide information about the time course of nanodrug accumulation and bioavailability in target organs}

Having established in vitro that targeting of miR-10b with $\mathrm{MN}$-anti-miR $10 \mathrm{~b}$ results in efficient cellular delivery, miRNA knockdown and inhibition of the invasion and migration of the tumor cells, we set out to determine if therapy could be delivered to primary and metastatic tumor cells in vivo. We treated mice bearing orthotopic human breast adenocarcinoma tumors with MN-anti-miR10b or an inactive nanodrug (MN-scr-miR). To assess the in vivo bioavailability of the nanodrug, we relied on its image-guidance properties. In-vivo MRI was used to obtain information about the time course of nanodrug delivery to tumor cells. ${ }^{9}, 18$ MRI revealed that the nanodrug was successfully delivered to the tumors, as shown by the decrease in transverse relaxation time (Figure 3a). Quantitative analysis suggested a tendency towards MN-anti-miR10b build-up in the tissue after the second treatment session (Figure 3b).

We also employed near infrared fluorescence optical imaging to study the whole-body bioavailability of the MN-anti-miR10b. The probe displayed uptake by the primary tumor as well as by the superficial brachial, inguinal and cervical lymph nodes, confirming that this is a suitable approach for combined tumor and lymph node delivery of the therapeutic agent (Figure 4a). The accumulation of the nanodrug in the primary tumor and lymph nodes was also confirmed by ex-vivo imaging (Figure 4b). Quantitative analysis indicated a significantly higher uptake of the nanodrug by the primary tumor and lymph nodes relative to muscle tissue (Figure 4c). We also evaluated the cellular distribution of the nanodrug histologically in frozen tumor and lymph node sections. Near infrared fluorescence represented the Cy5.5-labeled agent. It revealed extensive uptake of MN-anti-miR10b by the primary tumor cells (Figure 4d). As shown below, treatment with MN-anti-miR10b led to the inhibition of metastatic growth. Therefore, to show the nanodrug uptake in lymph nodes we used an inactive drug (MN-scr-miR). As expected, the nanodrug accumulated in both macrophages and lymph node metastases (Figure 4e). In the absence of metastatic tumor cells, the nanodrug distributed principally to macrophages (Supplementary Figure 2).

\section{MN-anti-miR10b can prevent metastasis}

The feasibility of preventing tumor cell dissemination from the primary tumor was assessed by treating tumor-bearing animals with $\mathrm{MN}$-anti-miR10b or MN-scr-miR beginning before the formation of detectable lymph node metastases. The expectation was that downregulation of the pro-metastatic miR-10b would elicit a therapeutic effect manifested as a reduction in metastatic burden., ${ }^{2,6}$ Nude mice were orthotopically implanted with the 
luciferase-expressing MDA-MB-231-luc-D3H2LN cell line, which metastasizes to the lymph nodes by 4 weeks after tumor implantation. Treatment with the nanodrug was initiated at 2 weeks after tumor implantation, before the formation of detectable lymph node metastases, and involved tail vein injections $(10 \mathrm{mg} / \mathrm{kg}$ of iron) delivered once a week for 4 weeks. In-vivo bioluminescence imaging was performed during each treatment and was used to visualize and quantify the metastatic burden from the luciferase-expressing cell line. It revealed that by the end of the treatment course, the signal in the experimental animals treated with MN-anti-miR10b was at pre-metastatic levels, indicating a prevention of tumor cell metastasis from the primary tumor to lymph nodes (Figure 5a). In contrast, there was visible dissemination of tumor cells to the lymph nodes of control animals treated with the MN-scr-miR. This therapeutic effect was highly reproducible (Figure 5b).

Histopathological analysis of the lymph nodes confirmed the absence of detectable metastases in animals treated with the nanodrug. Conversely, there was extensive invasion of the lymph nodes by tumor cells in control animals (injected with MN-scr-miR) accompanied by a disruption of lymph node architecture and the formation of reverse follicles (Figure 5c). These findings established that our nano-delivery method could successfully access the primary tumor and engage the tumor cell miRNA machinery in a sequence-specific way. The result is the effective prevention of metastasis.

\section{$\mathrm{MN}$-anti-miR10b can arrest the growth of lymph node metastasis}

In order to see if the nanotherapeutic method could alleviate metastatic disease once it had spread beyond the primary tumor and into the lymph nodes, we initiated treatment with MNanti-miR10b 4 weeks after tumor cell implantation, subsequent to the formation of lymph node metastases. As before, treatment with the nanodrug involved weekly intravenous injections ( $10 \mathrm{mg} / \mathrm{kg}$ of iron) for 4 weeks. As seen in Figures $6 \mathrm{a}$ and $\mathrm{b}$, there was a 20 -fold increase in lymph node metastatic burden in control animals treated with MN-scr-miR. By contrast, there was a relative arrest of metastastatic growth in the experimental animals treated with the active nanodrug, $\mathrm{MN}$-anti-miR10b, without a concomitant effect on primary tumor growth (Figure 6c; Supplementary Figure 3). This effect was accompanied by induction of the known miR-10b target HOXD $10^{6}$ at both sites (Supplementary Figure 4), indicating that the function of miR-10b in tumor cell migration is conserved between the primary and lymph node metastatic tumor cells.

Consequently, the relative arrest of metastasis by miR-10b inhibition was surprising. Metastatic burden is a result of both repopulation of the distant organ by cells from the primary tumor and proliferation of already metastasized tumor cells. In our studies, the absence of an effect on primary tumor growth suggested that miR-10b did not influence tumor cell proliferation or apoptosis. The latter observation is also supported by the prior literature. ${ }^{6}$ Therefore, the observed arrest of metastatic growth in the lymph nodes pointed to a context-dependent variation in miR-10b function. It implied that whereas in the primary tumors miR-10b only appeared to control metastatic dissemination, at the lymph node metastatic site it played a role in macroscopic tumor formation.

To investigate this possibility, we first surgically removed the primary tumor after the establishment of lymph node metastases and then initiated treatment with the nanodrug. This experiment would allow us to see whether the arrest of metastatic growth in lymph nodes in the $\mathrm{MN}$-anti-miR10b-treated mice was partly due to reduced tumor cell proliferation in the lymph nodes, independently of continued migration and invasion of cells from the primary tumor. After the completion of the treatment, we observed metastatic growth in the lymph nodes of MN-scr-miR-treated mice, but not in MN-anti-miR10b-treated mice (Supplementary Figure 5). 
To further investigate the possibility of a context-dependent miR-10b role in metastasis, we performed microRNA microarray analysis of the MN-anti-miR10b-treated MDA-MB-231luc-D3H2LN cells. Of the identified differentially expressed miRNAs (Supplementary Figure 6), miR-200c and miR-155 were notable for their coordinate downregulation with miR-10b, because both miRNAs have previously been validated as context-dependent players in metastasis. Namely, while in the primary tumors they serve as inhibitors of epithelial-to-mesenchymal transition and metastasis, at the distant site, they promote macroscopic tumor formation. ${ }^{19,20}$ Consequently, their coordinate downregulation with $\mathrm{miR}-10 \mathrm{~b}$ is in line with the hypothesis that miR-10b can participate not only in the regulation of tumor cell dissemination but also macroscopic tumor formation, depending on the specific tumor cell microenvironment.

Evidence about the specific mechanisms behind this context-dependent miR-10b role was derived from histological analysis of apoptosis and proliferation in the primary tumors vs lymph nodes of experimental and control animals. We observed no differences in the apoptotic rates of the primary tumor cells or lymph node metastases between experimental and control animals (Figure 7a; Supplementary Figure 7). However, in the presence of miR-10b inhibition, tumor cell proliferation was significantly reduced in the lymph node metastases but not in the primary tumor cells (Figure 7b; Supplementary Figure 7). This effect could account for the observed arrest of metastatic growth in the lymph nodes while growth of the primary tumor was still maintained.

Finally, as an important step towards establishing the feasibility of applying our nanodrug in a true therapeutic context, we assessed the liver toxicity of the agent. We measured aspartate aminotransferase and alanine aminotransferase levels in mice following treatment with the nanodrug and using phosphate-buffered saline (PBS) treatment, as control. The aspartate aminotransferase levels were 78.5 \pm 1.8 and $77.6 \pm 12.7$ (IU/1), in nanodrug and PBS-treated animals, respectively. The corresponding alanine aminotransferase levels were $17.9 \pm 0.4$ and $15.3 \pm 0.6$ (IU/l). These data indicate the absence of acute liver toxicity associated with the nanodrug.

\section{DISCUSSION}

The relevance of miRNA to human cancer became apparent just within the last decade. ${ }^{21}$ Since then, there has been a growing list of reports demonstrating the critical role played by miRNA in cancer initiation and progression. miRNAs represent a promising therapeutic target because their expression is uniquely altered in tumor cells and represents a 'hub' of carcinogenesis such that a single miRNA can coordinately affect the expression of multiple genes. In this sense, miRNAs represent molecular switches that can be 'turned on or off' to elicit a comprehensive therapeutic response.

The present study describes the application of nanotherapy targeting miRNA-10b for the prevention of metastatic breast cancer. Our findings also identify an unexpected effect manifested as arrest of metastatic growth in the lymph nodes, suggesting a contextdependent role for miR-10b in breast cancer metastasis that can be utilized to inhibit metastatic progression at the lymph node stage of the disease. In particular, inhibition of miR-10b mediated not only the prevention of metastatic spread from the primary tumor to lymph nodes but also the arrest of metastatic growth in the already formed lymph node metastases. A deeper investigation into the mechanisms behind this effect suggested context-dependent differences in miR-10b oncogenesis. Whereas in the primary tumor, miR-10b appeared to control metastasis but not tumor growth, in the lymph nodes, the role of the miRNA also extended to inhibition of tumor proliferation, which could be in part due to coordinate changes in the expression of miR-200c and miR-155. 
Such context-dependent differences in miRNA function have only begun to emerge. It is now known that a single miRNA can regulate the expression of multiple principal targets in a specific microenvironment. In different cellular contexts, the same miRNA may exhibit diverse functions, depending on the repertoire and stoichiometry of its direct mRNA targets. ${ }^{22}$ Specifically, whereas in breast cancer cells, miR-10b promotes invasion and metastasis through post-transcriptional regulation of HOXD10, in glioblastoma, miR-10b promotes proliferation and prevents death of cancer cells by targeting cell-cycle inhibitors and pro-apoptotic genes. ${ }^{23}$

While differences in miRNA function between different tumor types have been established for some time now, it is not until recently that context-dependent distinctions between primary and metastatic cells of the same tumor were identified. Notable examples include miR-200c and miR-155. Both microRNAs have a well-documented role as inhibitors of epithelial-to-mesenchymal transition and metastasis. However, whereas in mammary fat pads the two miRNAs prevent tumor dissemination, at the metastatic site apparently they maintain the epithelial phenotype of tumor cells that is critical for macroscopic tumor formation. ${ }^{19,20}$ Our studies now add miR-10b to the list of microRNAs that seemingly have different functions in different microenvironments and suggest a pleiotropic effect for the miRNA that involves both prevention of tumor cell dissemination and reduction of macroscopic tumor formation. This role of miR-10b may be specific to the lymph node metastatic site considering recent studies, which suggest that miR-10b does not influence the progression of already established lung metastasis, following intravenous injection of aggressive breast adenocarcinoma cells. ${ }^{6}$

Based on our findings and the recent literature, ${ }^{19,20,22}$ we expect that this context dependence reflects a fundamental property of the miRNA epigenome and extends to a variety of biological scenarios. Consequently, the tools described here could have a broad relevance for the study of microRNA function and its targeting for therapy.

Namely, the nanodrug utilizes a versatile platform suitable for conjugation to multiple moieties. Consequently, the nanodrug's modular design can be tailored for the establishment of a wide variety of 'therapeutic' molecular phenotypes in different organs and cell types. In addition, the nanodrug can be detected in a semi-quantitative manner by noninvasive imaging. This is advantageous because it can lead to the development of optimized drug delivery strategies and, importantly, guide the implementation of individualized treatment protocols.

The overall significance of the work extends from the fact that metastases, and not the primary tumors from which they derive, are the key reason for mortality from carcinoma (>90\%). Even in the case of successful removal of the primary tumor, a complete cure is uncertain. Many patients already harbor disseminated tumor cells when they initially present with cancer. In most instances, cancer therapy does not eradicate these cells and cancer can recur. Therefore, it becomes imperative to design not only methods for detecting these tumor cells but also to develop effective anti-metastatic therapies. ${ }^{1}$ This is especially true for breast cancer, which can reappear even if the cancer was confined to the breast at the time of detection. Once metastatic breast cancer has been diagnosed, treatments generally do not lead to long-term survival.

In this context, our studies hold clinical potential, because they would establish the feasibility of a new method for image-guided delivery of miRNA therapy. Considering that related $\mathrm{MN}$ are already in clinical use, including for detection and staging of breast cancer in patients, ${ }^{24,25}$ the technology developed in this report may ultimately become applicable in a 
clinical setting, advancing our ability to treat cancer at later stages of the pathology and impacting overall patient survival.

\section{MATERIALS AND METHODS}

\section{Locked nucleic acids}

The short LNA oligonucleotide sequence (anti-miR-10b), 5' -ThioMC6-D/

GTGTAACACGTCTATACGCCCA- $3^{\prime}$, directed against miRNA-10b and a mismatch scrambled sequence (scr-miR), 5' -ThioMC6-D/CACAAATTCGGTTCTACAGGGTA-3', were synthesized by Exiqon Inc. A $5^{\prime}$-Thiol modification was inserted into both sequences for conjugation to $\mathrm{MN}$ and a $3^{\prime}$-Cy3 modification was inserted in the sequences for in-vitro studies. The thiol modifications on the oligonucleotides were activated with treatment of $3 \%$ TCEP (Tris(2-carboxyethyl)phosphine hydrochloride), followed by purification with ammonium acetate/ethanol precipitation treatment before conjugation to the nanoparticles as described previously. ${ }^{8,9}$

\section{Synthesis of CRGD peptide}

The synthesis of Cyclic RGDfK-PEG-Cys-(Boc) was carried out as follows. To a solution of Boc-Cys(Tris)-Osu (11.2 mg, $0.02 \mathrm{mmol})$ in dimethylformamide (DMF) $(1 \mathrm{ml})$ was added cyclo [Arg-Gly-Asp-- -Phe-Lys(PEG-PEG) (where PEG=8-Amino-3,6-dioxaoctanoic acid) (13.41 mg, $0.015 \mathrm{mmol}$, Peptide International Inc., Louisville, KY, USA). The reaction mixture $\mathrm{pH}$ (8.5-9) was maintained using DIEA (diisopropyl ethylmine) and the resulting reaction mixture was allowed to stir at room temperature overnight. The product of the reaction was confirmed by TLC (thin layer chromatography) and isolated using the HPLC (high pressure liquid chromatography) gradient method. Collected fractions were lyophilized and obtained as a white powder. The final product was analyzed by MALDITOF (matrix-assisted laser desorption/ionization-time of flight) mass spectrometry.

Cyclic RGDfK-PEG-Cys-(Boc) was treated with 2-3 ml of anhydrous trifluoroacetic acid at room temperature for $30 \mathrm{~min}$. The resulting volatile mixture was completely removed under vacuum. Afterwards, the residue was dissolved in $100 \mathrm{~m}_{\mathrm{N}} \mathrm{NH}_{4} \mathrm{OAc}$ buffer $(3 \mathrm{ml})$. The resulting solution was filtered, and the filtrate was purified by HPLC. The final product was analyzed by MALDI-TOF Mass spectrometry.

\section{Synthesis of dextran-coated MN}

The MN synthesis was modified from a protocol published previously. ${ }^{26}$ Briefly, $9 \mathrm{~g}$ of Dextan-T10 (Pharmacosmos, Holbaek, Denmark) was dissolved in $30 \mathrm{ml}$ of double-distilled water and stirred in a round bottom flask on ice. $\mathrm{FeCl}_{3} \cdot 6 \mathrm{H}_{2} \mathrm{O}(0.65 \mathrm{~g})$ was added while flushing Argon gas into the reaction mixture for an hour. $\mathrm{FeCl}_{2} \cdot 4 \mathrm{H}_{2} \mathrm{O}(0.4 \mathrm{~g})$ was added into the mixture and then $15 \mathrm{ml}$ of concentrated cold $\mathrm{NH}_{4} \mathrm{OH}(\sim 28 \%)$ was added dropwise to the stirring mixture. The temperature increased to $85^{\circ} \mathrm{C}$ for an hour to induce the formation of a nanoparticulate colloidal mixture, cooled to room temperature and concentrated to $20 \mathrm{ml}$ using Amicon Ultra centrifugal units (MWCO $30 \mathrm{kDa}$; Millipore, Billerica, MA, USA). The resulting $20 \mathrm{ml}$ dextran-coated $\mathrm{MN}$ were cross-linked and aminated with subsequent addition of $35 \mathrm{ml}$ of $5 \mathrm{M} \mathrm{NaOH}, 14 \mathrm{ml}$ of concentrated epichlorohydrin $(8 \mathrm{~h})$ and $60 \mathrm{ml}$ of concentrated $\mathrm{NH}_{4} \mathrm{OH}$. The nanoparticle solution was purified using a dialysis bag (MWCO $14 \mathrm{kDa}$ ) against water and $20 \mathrm{mM}$ citrate buffer ( $\mathrm{pH} \mathrm{8.0)}$ and then concentrated to $20 \mathrm{ml}$ by Amicon Ultra centrifugal units. The nanoparticle concentration was determined based on iron concentration $(10.8 \mathrm{mg} / \mathrm{ml} \mathrm{Fe})$ and measured spectrophotometrically, as described. ${ }^{9}$ The size of the nanoparticles was determined by dynamic light scattering using Zetasizer Nano ZS (Malvern Instruments Ltd, Malvern, Worcestershire, UK) and nanoparticles of 
$23.5 \pm 3.5 \mathrm{~nm}$ were selected as suitable for accumulation in tumors and lymph nodes. The number of amine groups was found to be 73 per nanoparticle.

\section{Nanodrug synthesis and characterization}

Briefly, $1 \mathrm{mg}$ of NIR dye Cy5.5 mono-reactive NHS ester (GE Healthcare, Piscataway, NJ, USA) was dissolved in $100 \mu$ l of anhydrous DMSO and incubated with MN (10 mg Fe) in $20 \mathrm{mM}$ citrate buffer ( $\mathrm{pH}$ 8.0) overnight. The nanoparticles were purified using Sephadex PD-10 column (GE Healthcare) against PBS. The number of Cy5.5 molecules per nanoparticle was quantified spectrophotometrically and found to be four Cy5.5 molecules per MN. The nanoparticles were further conjugated to heterofunctional linker SPDP $(\mathrm{N}-$ succinimidyl 3-[2-pyridyldithio]-propionate; Pierce Biotechnology, Rockford, IL, USA) in order to provide a thiol reactive terminus for LNA and cRGD conjugation. Briefly, $10 \mathrm{mg}$ SPDP was dissolved in $500 \mu \mathrm{l}$ anhydrous DMSO and incubated with Cy5.5-labeled MN. The MN were further conjugated to cRGD peptide through its cysteine terminus in PBS and purified with a Sephadex PD-10 column. The LNA oligos were then conjugated to MN. Briefly, the thiolated $5^{\prime}$ terminus of the oligo was activated via 3\% TCEP treatment in nuclease free PBS. The LNA oligos were purified using ammonium acetate/ethanol precipitation method. After TCEP-activation and purification, the oligos were resuspended in PBS and $50 \mathrm{~m}_{\mathrm{M}}$ EDTA and incubated with the nanoparticles overnight. The resulting probe was purified using a G-50 Sephadex disposable quick spin columns (Roche Applied Science, Indianapolis, IN, USA). The quantification of cRGD and LNA per MN was described previously ${ }^{9}$ and determined as 15 cRGD per MN and 10 LNA per MN.

\section{Cell lines}

We maintained MDA-MB-231-GFP (kindly provided by Dr Danny R Welch, University of Alabama at Birmingham), 4T1 (American Type Culture Collection (ATCC), Manassas, VA, USA) and MDA-MB-231-luc-D3H2LN (Caliper Life Sciences, Hopkinton, MA, USA) metastatic breast cancer cell lines as recommended by the supplier.

\section{Fluorescence confocal microscopy}

Fluorescence confocal microscopy was performed on MDA-MB-231-GFP breast cancer cells. Cells $\left(2 \times 10^{6}\right)$ were incubated with MN-anti-miR10b or MN-scr-miR $(45 \mu \mathrm{g} \mathrm{Fe}, 4$ nmol LNA) for $48 \mathrm{~h}$ at $37{ }^{\circ} \mathrm{C}$ on a cover slip in an eight-well plate. The cells were then washed three times with Hank's buffered salt solution (HBSS) buffer and fixed with $2 \%$ formaldehyde for $10 \mathrm{~min}$. The cells were then washed three times with DPBS buffer and the cover slip was placed on a glass slide with Vectashield mounting medium (Vector Laboratories, Burlingame, CA, USA). The slides were dried in the hood for $30 \mathrm{~min}$ in a dark room. The cells were imaged by confocal microscopy in the FITC channel (GFP detection), the Cy3 channel (LNA detection) and the Cy5.5 channel (MN detection) using a Zeiss LSM 5 Pascal laser confocal microscope (Carl Zeiss Microscopy, Thornwood, NY, USA). The Zeiss RGB vario laser module consists of an argon laser (458/488/514 nm) and two heliumneon lasers (543 and $633 \mathrm{~nm}$ ). Image acquisition and analyses were performed using Zeiss LSM 5 Pascal Confocal Microscopy Software (Release 3.2). The final images were colorcoded green for GFP (cells), red for Cy3 (LNA oligos) and blue for Cy5.5 (MN).

\section{Flow cytometry}

MN-anti-miR10b and MN-scr-miR nanodrug uptake by MDA-MB-231-GFP and 4T1 cells was analyzed by flow cytometry. MDA-MB-231-GFP cells were incubated with the probes ( $45 \mu \mathrm{g} \mathrm{Fe}, 4 \mathrm{nmol} \mathrm{LNA}$ ) for $48 \mathrm{~h}$ and $4 \mathrm{~T} 1$ cells were incubated with the probes $(45 \mu \mathrm{g} \mathrm{Fe}, 4$ nmol LNA) for $24 \mathrm{~h}$ at $37^{\circ} \mathrm{C}$ in an eight-well plate, washed twice with HBBS buffer and removed from the plate using Hank's-based enzyme free cell dissociation buffer. The cells 
were then fixed in $2 \%$ paraformaldehyde for $1 \mathrm{~h}$ at $4{ }^{\circ} \mathrm{C}$ and diluted in sheath solution for flow cytometry measurements. Nanodrug uptake was analyzed in the FL4 channel (Cy5.5, MN) and FL2 channel (Cy3, LNA oligos) using FACSCalibur (Becton Dickinson, Franklin Lakes, NJ, USA) equipped with the CellQuest software package (Franklin Lakes, NJ, USA).

\section{Real-time quantitative reverse transcription-PCR}

To measure the extent of miR-10b knockdown by the nanodrug, MDA-MB-231 and 4T1 cells were incubated with MN-anti-miR10b and MN-scr-miR ( $45 \mu \mathrm{g}, 4 \mathrm{nmol} L N A)$ for $48 \mathrm{~h}$ at $37^{\circ} \mathrm{C}$. The miRNA-enriched fraction from total extracted RNA was harvested using the miRNeasy mini kit, according to the manufacturer's protocol (Qiagen Inc, Valencia, CA, USA). Relative levels of miR-10b were determined by real-time quantitative reverse transcription-PCR (qRT-PCR; Taqman protocol) and compared with the internal housekeeping gene SNORD44. Taqman analysis was carried out using an ABI Prism 7700 sequence detection system (PE Applied Biosystems, Foster City, CA, USA). The primers were provided by the manufacturer ( $\mathrm{RT}^{2}$ miRNA First Strand Kit, miRNA qPCR assay for hsa-miR-10b and SNORD44 as endogenous control; SABiosciences).

MicroRNA microarray analysis was performed using the human cancer microRNA PCR array according to the manufacturer's instructions (MAH-102A, SABiosciences, Valencia, CA, USA). Data were analyzed using the WEB-based PCR array data analysis software provided by the manufacturer.

\section{Western blot}

Frozen tumor sections were thawed and homogenized in tissue protein extraction lysis buffer (Tissue-PE LB from G-Biosciences, St Louis, MO, USA) along with $1 \mathrm{~m}_{\mathrm{M}}$ PMSF and proteinase inhibitor cocktails (Sigma, St Louis, MO, USA). Protein content was determined with the Bio-Rad protein assay kit (Bio-Rad, Hercules, CA, USA). Lysates $(50 \mu \mathrm{g})$ were separated by electrophoresis through 4-20\% sodium dodecyl sulfate polyacrylamide gel electrophoresis and transferred to nitrocellulose membranes (Bio-Rad). Membranes were blocked in 5\% nonfat milk in Tris-buffered saline/Tween 20 for $1 \mathrm{~h}$ at room temperature. After blocking, the membrane was incubated overnight at $4{ }^{\circ} \mathrm{C}$ in $1 \%$ milk/TBS containing HOXD10 (H-80) rabbit polyclonal Antibody (1:200, Santa Cruz Biotechnology, Santa Cruz, CA, USA) and mouse monoclonal $\beta$-actin $(1 \mu \mathrm{g} / \mathrm{ml}$; Applied Biosystems, Carlsbad, CA, USA). The membrane was then washed three times with $0.05 \%$ Tween TBS (TBST) for 5 min each and incubated with horseradish peroxidase-conjugated goat anti-rabbit or antimouse antibodies (Invitrogen, Camarillo, CA, USA) (1:2000 dilution) for $60 \mathrm{~min}$ at room temperature, followed by washing three times with TBST and one time with TBS for $5 \mathrm{~min}$ each. Membranes were finally developed using ECL plus western blotting detection reagents kit (GE Healthcare), according to the manufacturer's specifications.

\section{In-vitro migration and invasion assays}

Assays were performed according to the supplier's instructions (Cell Biolabs, San Diego, CA, USA). Briefly, MDA-MB-231 $\left(0.1 \times 10^{6}\right)$ cells were plated in each insert containing membrane (pore size $8 \mu \mathrm{m}$ ) and corresponding nanodrug (60 $\mu \mathrm{g}, 5.0 \mathrm{nmol}$ LNA) was added with/without $10 \%$ FCS (fetal calf serum) and incubated for $24 \mathrm{~h}$ for migration and $48 \mathrm{~h}$ for invasion assay. The inserts were stained with the supplier's kit and membranes are imaged by light microscopy to score migration and invasion.

\section{Animal models}

Six-week-old female nude mice (nu/nu or NIH III nude) were implanted orthotopically with the human breast adenocarcinoma MDA-MB-231-luc-D3H2LN cell line (Caliper Life 
Sciences). In this model, orthotopically implanted tumors progress from localized disease to lymph node metastasis by 4 weeks after tumor inoculation. The tumor cells express luciferase and can be detected by noninvasive bioluminescence imaging for correlative analysis of tumor burden. All animal experiments were performed in compliance with institutional guidelines and approved by the Subcommittee on Research Animal Care (SRAC) at Massachusetts General Hospital.

\section{Prevention of metastasis}

Six week-old $n u / n u$ mice ( $n=12$; Massachusetts General Hospital Radiation Oncology breeding facilities) were injected in the upper right mammary fat pad with $2 \times 10^{6}$ MDAMB-231-luc-D3H2LN cells (Caliper). Animals were used in experiments 14 days after tumor implantation.

\section{Arrest of metastasis}

Six-week-old NIH III nude mice ( $n$ 6; Massachusetts General Hospital Radiation Oncology breeding facilities) were injected in the lower left mammary fat pad with $2 \times 10^{6} \mathrm{MDA}-$ MB-231-luc-D3H2LN cells (Caliper). Animals were used in experiments 28 days after tumor implantation.

Treatment with $\mathrm{MN}$-anti-miR10b and $\mathrm{MN}$-scr-miR involved systematic administration through the tail vein at a dose of $10 \mathrm{mg} \mathrm{Fe} / \mathrm{kg}$ once a week over 4 weeks.

\section{Arrest of metastasis in mice with surgically removed primary tumor}

Six-week-old nu/nu mice ( $n=6$; Massachusetts General Hospital Radiation Oncology breeding facilities) were injected in the lower left mammary fat pad with $2 \times 10^{6}$ MDAMB-231-luc-D3H2LN cells (Caliper). Tumors were surgically removed 28 days after tumor implantation.

Treatment with $\mathrm{MN}$-anti-miR10b and $\mathrm{MN}$-scr-miR involved systematic administration through the tail vein at a dose of $10 \mathrm{mg} \mathrm{Fe} / \mathrm{kg}$ once a week over 4 weeks.

\section{In-vivo MRI}

MRI was performed before and $24 \mathrm{~h}$ after intravenous administration of the nanodrug using a 9.4 T Bruker horizontal bore scanner with ParaVision 5.1 software (Bruker Biospin, Billerica, MA, USA). The imaging protocol consisted of coronal T2-weighted spin echo pulse sequences with the following parameters: spin echo repetition time/echo time $=2000$ / $[8,16,24,32,40,48,56,64]$; field of view $(\mathrm{FOV})=32 \times 34 \mu \mathrm{m}^{2} ;$ matrix size $=128 \times 128$ pixels; slice thickness $=0.5 \mathrm{~mm}$; in plane resolution $=250 \times 250 \mu \mathrm{m}^{2}$. Images were reconstructed and analyzed by Marevisi 3.5 software (Institute for Biodiagnostics, National Research Council, Canada). T2 maps were constructed according to established protocol by fitting $\mathrm{T} 2$ readings for each of the eight echo times to a standard exponential decay curve.

T2 relaxation times were calculated by manually segmenting out the tumor on MR images from each slice for every animal before and after nanodrug injection. Longitudinal relaxation rate $(R 2=1 / T 2)$ was determined for each slice and $\Delta R 2$ was calculated by subtracting the $\mathrm{R} 2$ readings before from those after nanodrug administration.

\section{In-vivo and ex-vivo optical imaging}

In-vivo fluorescence optical imaging was performed immediately after each in-vivo MR imaging session. Anesthetized animals were placed supine into a whole-body imaging system (IVIS Spectrum, Caliper Life Sciences) equipped with 10 narrow band excitation 
filters ( $30 \mathrm{~nm}$ bandwith) and 18 narrow band emission filters (20 $\mathrm{nm}$ bandwith) that assist in significantly reducing autofluorescence through spectral scanning of filters and the use of spectral unmixing algorithms (Caliper Life Sciences). The abdominal region of the animal was shielded in order to avoid interfering signal from internal organs. Imaging was performed using 675-nm excitation and 720-nm emission filters. The epifluorescent images and the grayscale photographs were acquired and superimposed.

EX-vivo fluorescent images were acquired by placing the excised tissues in the imaging system immediately after the animals were killed. The average radiance from each fluorescent reading was used to estimate nanodrug uptake. The images were reconstructed using Living Image software version 4.0 (Caliper Life Sciences).

\section{In-vivo optical bioluminescence imaging}

Mice were injected in the lower left abdominal quadrant with ${ }_{\mathrm{D}}$-Luciferin potassium salt firefly in DPBS (150 mg Luciferin/kg body weight, $200 \mu 1 \mathrm{of} 15 \mathrm{mg} / \mathrm{ml}$; Caliper Life Sciences) 10 min before image acquisition. The primary tumor was shielded to prevent signal leakage into the right brachial lymph node. Identical imaging settings (time, 30-60 s; F-stop, 2; binning, medium) and same-size regions of interest were used to obtain total radiance flux of the metastatic signals from right brachial lymph nodes. The total radiance (photons/s) from the bioluminescent readings was used for signal quantification.

For the study on metastatic arrest, the lower abdominal primary tumor in the mammary fat pad was shielded and the total bioluminescence flux reading (photons/s) was taken from the right brachial lymph node with a fixed region of interest (F-stop, 8; binning large). Relative tumor and metastatic burdens were calculated as fold change in bioluminescence flux (photons/s) from the tumor or lymph node regions of interest relative to the first week of treatment.

\section{Histology and fluorescence microscopy of tissue sections}

To detect the metastatic lesions and/or the accumulation of the nanodrug in the primary tumor and lymph nodes, the excised tissues were embedded in Tissue-Tek OCT compound (Sakura Finetek) and snap frozen in liquid nitrogen. The frozen primary tumor and lymph nodes were cut into $7 \mu \mathrm{m}$ sections. The sections were fixed in $2 \%$ paraformaldehyde, washed and counterstained with Vectashield mounting medium containing 4',6-diamidino-2phenylindole (DAPI; Vector Laboratories) and analyzed by fluorescence microscopy using a Nikon Eclipse 50i fluorescence microscope equipped with the necessary filter sets. Images were acquired using a charge coupled device camera with near-IR sensitivity (SPOT 7.4 Slider RTKE; Diagnostic Instruments, Sterling Heights, MI, USA). The images were analyzed using SPOT 4.0 Advance version software (Diagnostic Instruments). The observed Cy5.5 signal within tumor sections was the result of accumulation of the intravenously injected nanodrug.

For macrophage staining, frozen lymph node sections were incubated with a rat anti-mouse CD68 (FA-11) antibody (AbD Serotec, Raleigh, NC, USA) followed by a rhodaminelabeled goat secondary antibody (Abcam, Cambridge, UK). Final images were color-coded blue for DAPI (nuclei), red for Cy5.5 (nanodrug) and green for rhodamine (macrophages). Consecutive tissue sections were stained with hematoxylin and eosin (H\&E) and analyzed by light microscopy to determine metastatic burden or to compare fluorescent images with H\&E-defined tissue architecture.

Apoptosis in tumor tissues was evaluated by performing a terminal deoxynucleotidyl transferase-mediated dUTP nick end labeling assay (ApopTag Fluorescein In situ Apoptosis 
Detection kit, Chemicon International, Billerica, MA, USA) according to the manufacturer's protocol.

For determining proliferation, frozen tumor and lymph node sections were incubated with a rabbit anti-Ki67 antibody (Abcam) followed by a DyLight-488-labeled goat secondary antibody (Abcam).

\section{Liver toxicity}

Liver toxicity following the treatment with the nanodrug was determined by monitoring aspartate aminotransferase and alanine aminotransferase levels, using commercially available kits (Pointe Scientific, Canton, MI, USA), according to the manufacturer's protocol.

\section{Statistical analysis}

Data were expressed as mean \pm s.d. or s.e.m., where indicated. Statistical differences between experimental and control groups were analyzed by a two-tailed $t$-test (SigmaStat 3.0; Systat Software, Richmond, CA, USA). Time-course data were analyzed by analysis of variance. A value of $P<0.05$ was taken as statistically significant.

\section{Supplementary Material}

Refer to Web version on PubMed Central for supplementary material.

\section{Acknowledgments}

We thank Pamela Pantazopoulos and Alana Ross for help with the in-vitro, in-vivo and ex-vivo studies and Marytheresa Ifediba for proofreading the manuscript. Confocal microscopy was performed at the Confocal Microscopy Core at MGH with technical assistance from Igor A Bagayev, MS Work at the Martinos Center for Biomedical Imaging was supported in part under Grant R00CA129070 from the National Cancer Institute and a Research Grant from the Breast Cancer Alliance.

\section{References}

1. Chaffer CL, Weinberg RA. A perspective on cancer cell metastasis. Science. 2011; 331:1559-1564. [PubMed: 21436443]

2. Ma L, Teruya-Feldstein J, Weinberg RA. Tumour invasion and metastasis initiated by microRNA-10b in breast cancer. Nature. 2007; 449:682-688. [PubMed: 17898713]

3. Ma L, Weinberg RA. Micromanagers of malignancy: role of microRNAs in regulating metastasis. Trends Genet. 2008; 24:448-456. [PubMed: 18674843]

4. Ma L, Weinberg RA. MicroRNAs in malignant progression. Cell Cycle. 2008; 7:570-572. [PubMed: 18256538]

5. Ma L, Young J, Prabhala H, Pan E, Mestdagh P, Muth D, et al. miR-9, a MYC/MYCN-activated microRNA, regulates E-cadherin and cancer metastasis. Nat Cell Biol. 2010; 12:247-256. [PubMed: 20173740]

6. Ma L, Reinhardt F, Pan E, Soutschek J, Bhat B, Marcusson EG. Therapeutic silencing of miR-10b inhibits metastasis in a mouse mammary tumor model. Nat Biotech. 2010; 28:341-347.

7. Baffa R, Fassan M, Volinia S, O'Hara B, Liu CG, Palazzo JP. MicroRNA expression profiling of human metastatic cancers identifies cancer gene targets. J Pathol. 2009; 219:214-221. [PubMed: 19593777]

8. Medarova Z, Pham W, Farrar C, Petkova V, Moore A. In-vivo imaging of siRNA delivery and silencing in tumors. Nat Med. 2007; 13:372-377. [PubMed: 17322898]

9. Kumar M, Yigit M, Dai G, Moore A, Medarova Z. Image-guided breast tumor therapy using a small interfering RNA nanodrug. Cancer Res. 2010; 70:7553-7561. [PubMed: 20702603] 
10. Zhao Y, Bachelier R, Treilleux I, Pujuguet P, Peyruchaud O, Baron R, et al. Tumor alphavbeta3 integrin is a therapeutic target for breast cancer bone metastases. Cancer Res. 2007; 67:58215830. [PubMed: 17575150]

11. Beer AJ, Niemeyer M, Carlsen J, Sarbia M, Nahrig J, Watzlowik P, et al. Patterns of alphavbeta3 expression in primary and metastatic human breast cancer as shown by $18 \mathrm{~F}-$ Galacto-RGD PET. $\mathrm{J}$ Nucl Med. 2008; 49:255-259. [PubMed: 18199623]

12. Kenny LM, Coombes RC, Oulie I, Contractor KB, Miller M, Spinks TJ, et al. Phase I trial of the positron-emitting Arg-Gly-Asp (RGD) peptide radioligand 18F-AH111585 in breast cancer patients. J Nucl Med. 2008; 49:879-886. [PubMed: 18483090]

13. Petersen M, Wengel J. LNA: a versatile tool for therapeutics and genomics. Trends Biotechnol. 2003; 21:74-81. [PubMed: 12573856]

14. Jepsen JS, Sorensen MD, Wengel J. Locked nucleic acid: a potent nucleic acid analog in therapeutics and biotechnology. Oligonucleotides. 2004; 14:130-146. [PubMed: 15294076]

15. Elmen J, Lindow M, Schutz S, Lawrence M, Petri A, Obad S, et al. LNA-mediated microRNA silencing in non-human primates. Nature. 2008; 452:896-899. [PubMed: 18368051]

16. Weissleder R, Elizondo G, Wittenberg J, Lee AS, Josephson L, Brady TJ. Ultrasmall superparamagnetic iron oxide: an intravenous contrast agent for assessing lymph nodes with MR imaging. Radiology. 1990; 175:494-498. [PubMed: 2326475]

17. Nathanson SD, Kwon D, Kapke A, Alford SH, Chitale D. The role of lymph node metastasis in the systemic dissemination of breast cancer. Ann Surg Oncol. 2009; 16:3396-3405. [PubMed: 19657697]

18. Medarova Z, Rashkovetsky L, Pantazopoulos P, Moore A. Multiparametric monitoring of tumor response to chemotherapy by noninvasive imaging. Cancer Res. 2009; 69:1182-1189. [PubMed: 19141648]

19. Xiang X, Zhuang X, Ju S, Zhang S, Jiang H, Mu J, et al. miR-155 promotes macroscopic tumor formation yet inhibits tumor dissemination from mammary fat pads to the lung by preventing EMT. Oncogene. 2011; 30:3440-3453. [PubMed: 21460854]

20. Dykxhoorn DM, Wu Y, Xie H, Yu F, Lal A, Petrocca F, et al. miR-200 enhances mouse breast cancer cell colonization to form distant metastases. PLoS One. 2009; 4:e7181. [PubMed: 19787069]

21. Calin GA, Dumitru CD, Shimizu M, Bichi R, Zupo S, Noch E, et al. Frequent deletions and downregulation of micro- RNA genes miR15 and miR16 at 13q14 in chronic lymphocytic leukemia. Proc Natl Acad Sci USA. 2002; 99:15524-15529. [PubMed: 12434020]

22. Gabriely G, Teplyuk NM, Krichevsky AM. Context effect: microRNA-10b in cancer cell proliferation, spread and death. Autophagy. 2011; 7:1-3.

23. Gabriely G, Yi M, Narayan RS, Niers JM, Wurdinger T, Imitola J, et al. Human glioma growth is controlled by microRNA-10b. Cancer Res. 2011; 71:3563-3572. [PubMed: 21471404]

24. Michel SC, Keller TM, Frohlich JM, Fink D, Caduff R, Seifert B, et al. Preoperative breast cancer staging: MR imaging of the axilla with ultrasmall superparamagnetic iron oxide enhancement. Radiology. 2002; 225:527-536. [PubMed: 12409591]

25. Harisinghani MG, Barentsz J, Hahn PF, Deserno WM, Tabatabaei S, van de Kaa CH, et al. Noninvasive detection of clinically occult lymph-node metastases in prostate cancer. $\mathrm{N}$ Engl J Med. 2003; 348:2491-2499. [PubMed: 12815134]

26. Medarova Z, Evgenov NV, Dai G, Bonner-Weir S, Moore A. In vivo multimodal imaging of transplanted pancreatic islets. Nat Protocols. 2006; 1:429-435. 

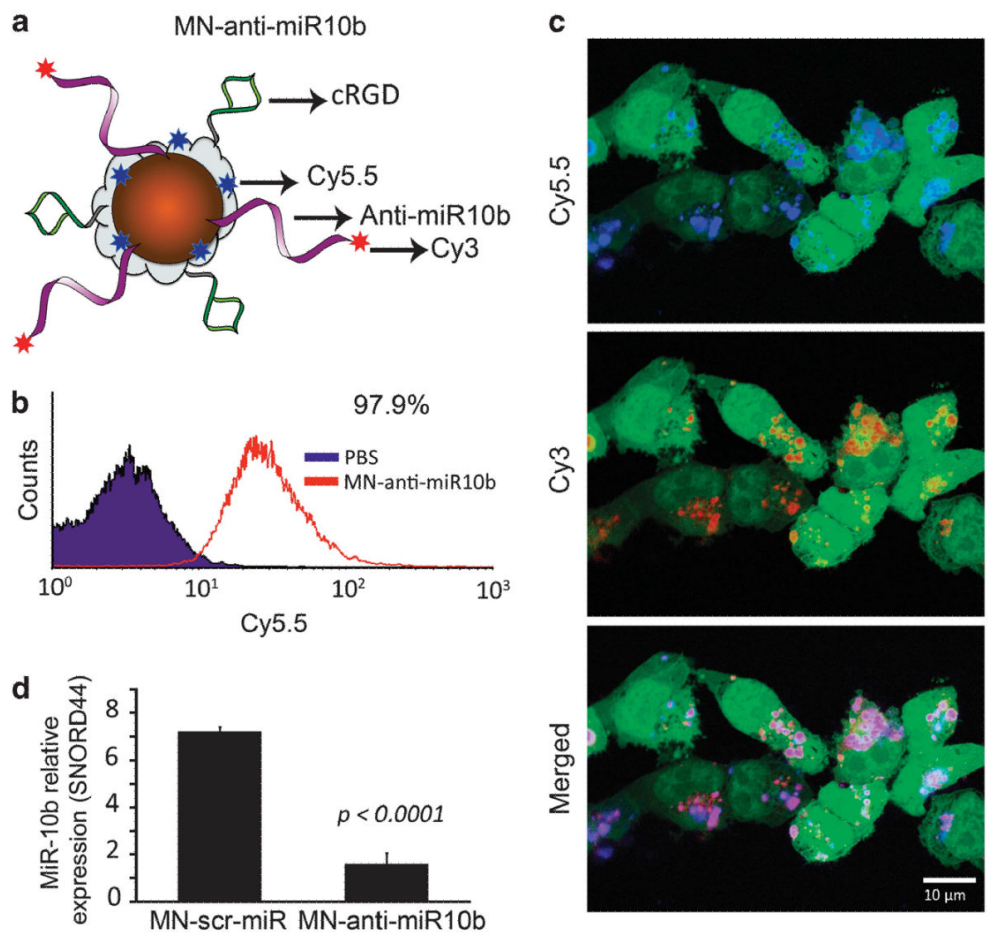

Figure 1.

In-vitro testing of nanodrug uptake, silencing efficacy and cytotoxicity in MDA-MB-231GFP human breast adenocarcinoma cells. (a) A schematic of the active nanodrug (MN-antimiR10b). The nanodrug consists of dextran-coated MN conjugated to Cy5.5 dye, a tumortargeting peptide (cRGD) and a knockdown LNA oligonucleotide targeting human miRNA-10b. (b) Flow cytometry, following a 48-h incubation with the nanodrug, demonstrating that nearly $100 \%$ of cells took up the probe. (c) Confocal microscopy of MDA-MB-231-GFP cells (green), following a 48-h incubation with the nanodrug, showing the distribution of the $\mathrm{MN}$ (Cy5.5, blue) and the LNA oligonucleotide (Cy3, red) components of the nanodrug. (d) qRT-PCR showing that the nanodrug mediated a significant $87.8 \pm 6.2 \%(P<0.0001, n=3)$ knockdown of the target miR-10b. Data are represented as mean \pm s.d. 
a

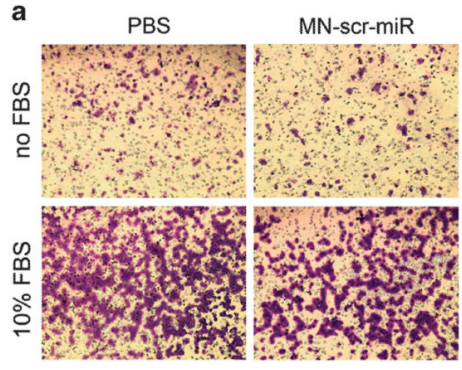

PBS
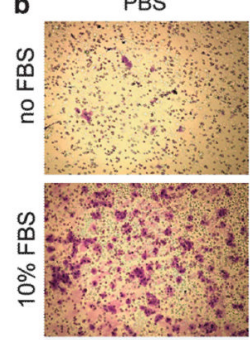

MN-scr-miR
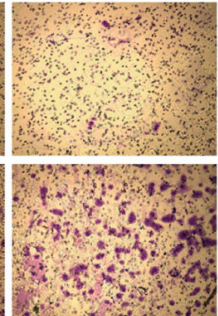

MN-anti-miR10b

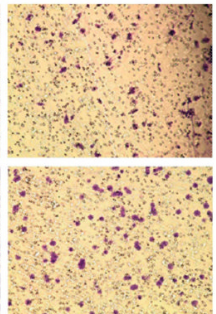

MN-anti-miR10b

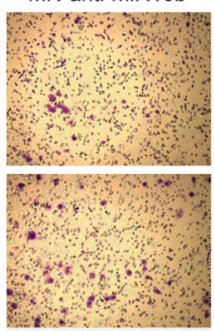

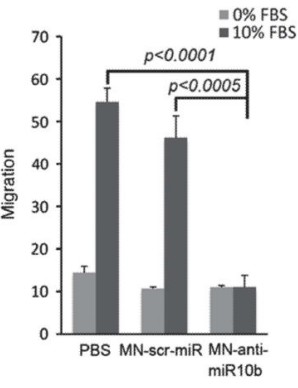

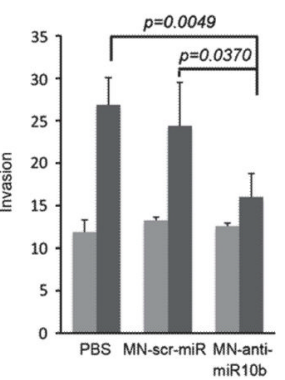

Figure 2.

Migration and invasion of MDA-MB-231-GFP cells treated with the active nanodrug, MNanti-miR $10 \mathrm{~b}$ or control probes. (a) Cell migration was significantly reduced by treatment with the nanodrug. The fraction of migrating cells (purple) in the presence of 10\% FBS was comparable to that in the absence of the migration stimulus (no FBS). This effect was not observed in the control groups $(n=3)$. (b) Cell invasion was significantly reduced by treatment with the nanodrug. The fraction of invasive cells (purple) in the presence of $10 \%$ FBS was comparable to that in the absence of the invasion stimulus (no FBS). This effect was not seen in the control groups $(n=3)$. 

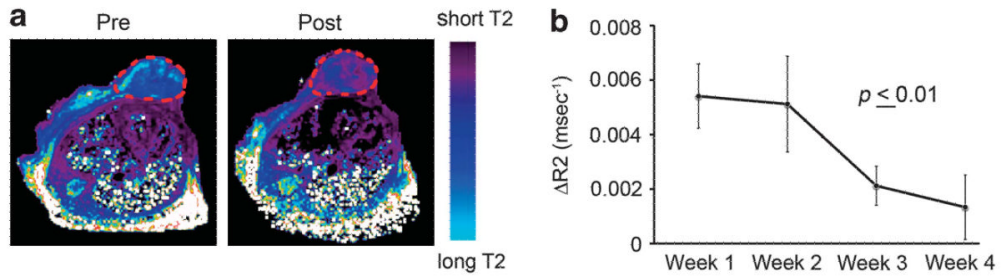

Figure 3.

T2-weighted MRI of MN-anti-miR10b accumulation in orthotopic MDA-MB-231-lucD3H2LN tumors. (a) Representative color-coded T2 maps before (left) and $24 \mathrm{~h}$ after (right) $\mathrm{MN}$-anti-miR10b injection, demonstrating a shortening of the $\mathrm{T} 2$ relaxation times of the tumors (outlined) consistent with nanodrug accumulation. (b) Quantitative analysis of $\Delta \mathrm{R} 2$ relaxation rates $(1 / \mathrm{T} 2$ pre- $1 / \mathrm{T} 2$ post, $\mathrm{ms})$ of the tumors, suggesting a tendency towards build-up of the MN-anti-miR10b $(P \unlhd) .01, n=12)$. Data are represented as mean \pm s.d. 

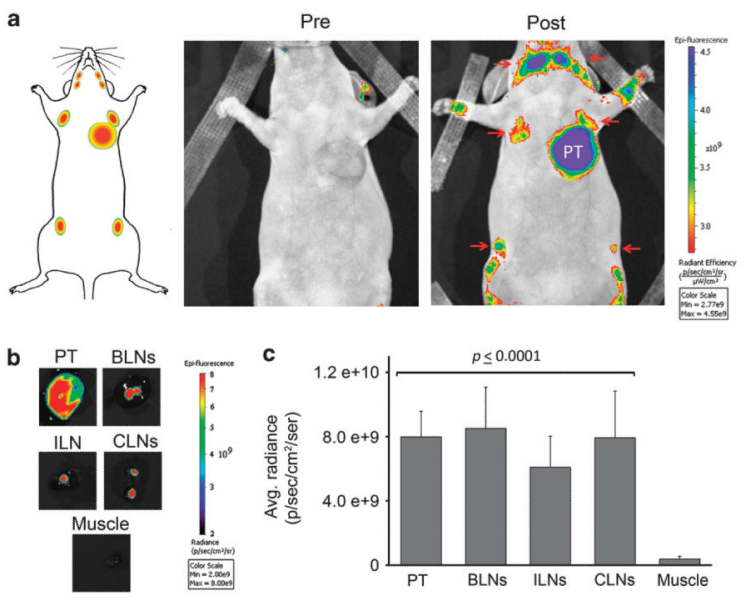

d

H\&E
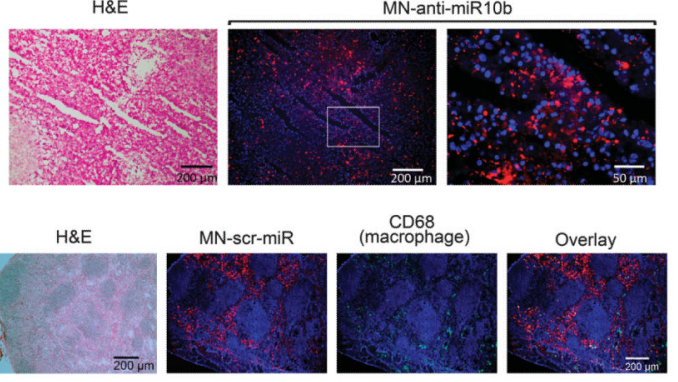

Figure 4.

NIR optical imaging of MN-anti-miR10b accumulation in orthotopic MDA-MB-231-lucD3H2LN tumors (a) In-vivo imaging of mice before (left) and $24 \mathrm{~h}$ after (right) MN-antimiR10b administration showing accumulation of the nanodrug in the primary tumor and lymph nodes. Arrows point to lymph nodes. (b) Ex-vivo images of excised tissues, demonstrating MN-anti-miR10b accumulation in the tumor tissues and lymph nodes (PT, primary tumor; BLNs, brachial lymph nodes; ILN, inguinal lymph nodes; CLNs, cervical lymph nodes). (c) Quantitative analysis of the ex-vivo optical images showing preferential uptake of the MN-anti-miR10b by the primary tumor and lymph nodes relative to muscle tissue, which is traditionally used as a reference $(P \unlhd 0.0001, n=6)$. Data are represented as mean \pm s.d. (d) Histology of frozen tumor sections. There was extensive uptake of the MNanti-miR10b by tumor cells. Red (Cy5.5, nanodrug), blue (DAPI, nuclei). (e) Histology of frozen lymph node sections from mice injected with MN-scr-miR (inactive drug that permits the formation of lymph node metastases). There was extensive uptake of the nanodrug not only by macrophages but also by tumor cells. Red (Cy5.5, nanodrug), blue (DAPI, nuclei), green (CD68, macrophages). H\&E: hematoxylin and eosin staining. 

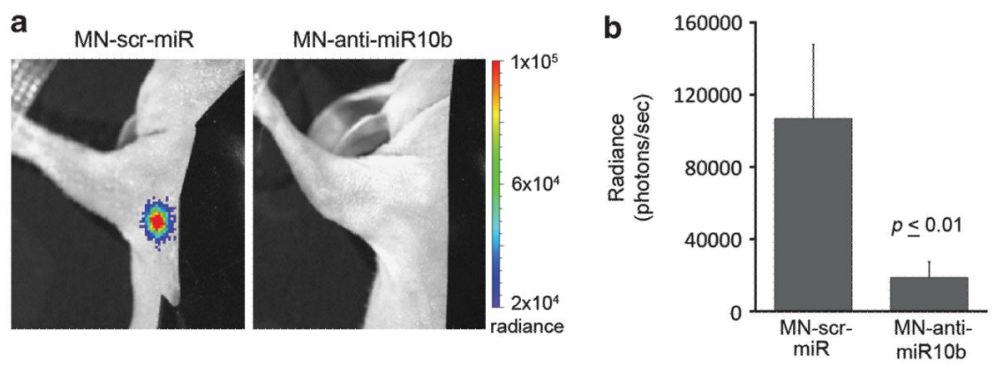

C

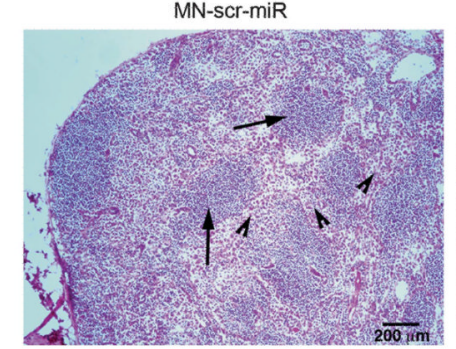

MN-anti-miR10b

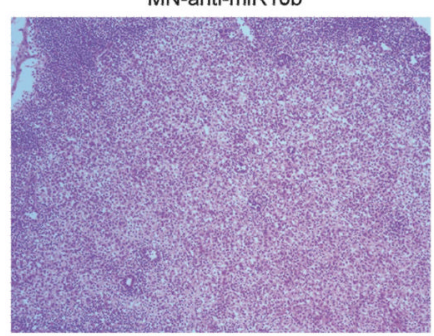

Figure 5.

Prevention of lymph node metastasis by MN-anti-miR10b. (a) Representative bioluminescence images of tumor-bearing mice treated with the nanodrug for 4 weeks beginning before lymph node metastasis. The mice were treated either with the active MNanti-miR10b or with inactive control MN-scr-miR. Whereas bioluminescence signal was visible in the brachial lymph nodes of control animals, bioluminescence in the experimental animals was at pre-metastatic levels, indicating prevention of metastasis by MN-antimiR10b. (b) Quantitative analysis of bioluminescence images. Radiance (photons/s) in the brachial lymph nodes of control mice treated with MN-scr-miR was significantly higher than in experimental animals treated with MN-anti-miR10b. In the experimental animals, radiance was at premetastatic levels indicating prevention of metastasis by MN-anti-miR10b $(P \unlhd 0.01, n=6)$. Data are represented as mean \pm s.e.m. (c) H\&E staining of frozen lymph node sections from animals treated with MN-anti-miR10b (right) or MN-scr-miR (left). In the control animals, there was extensive tumor cell infiltration (arrowheads) outside of reverse follicles (arrows). In animals treated with MN-anti-miR10b, lymph nodes had normal tissue architecture. 

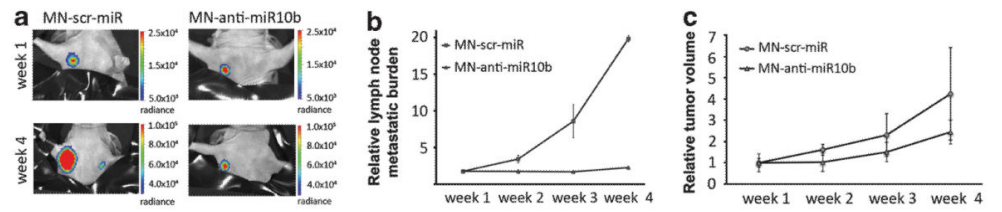

Figure 6.

Arrest of lymph node metastasis by MN-anti-miR10b. (a) Representative bioluminescence images of tumor-bearing mice treated with the nanodrug for 4 weeks beginning subsequent to lymph node metastasis. The mice were treated either with $\mathrm{MN}$-anti-miR10b or with inactive control MN-scr-miR. By the end of the study, there was a dramatic increase in bioluminescence signal from the brachial lymph nodes of control mice, whereas in the experimental mice the images suggested relative metastatic arrest. (b) Quantitative analysis. In the control animals, there was a 20 -fold increase in bioluminescence signal from the brachial lymph nodes. By contrast, bioluminescence in the experimental animals remained near pre-treatment levels, indicating that $\mathrm{MN}$-anti-miR10b could arrest metastasis in vivo $(P \unlhd 0.001, n=6)$. (c) Quantitative analysis of bioluminescence flux (photons/s) from the tumor regions of interest. There was no significant difference between experimental and control animals, indicating a lack of miR-10b influence on primary tumor growth $(n=6)$. Data are represented as mean \pm s.e.m. 

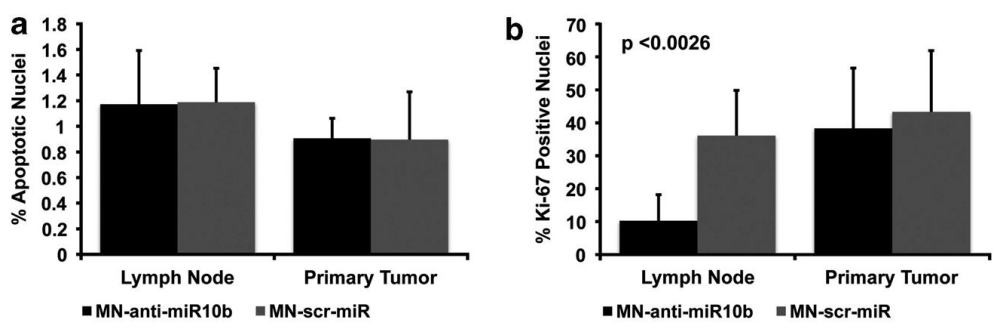

Figure 7.

Histological analysis of apoptosis and proliferation in the primary tumors vs lymph nodes of experimental and control animals. (a) Apoptotic rates in the primary tumors and lymph node metastases. The mice were treated either with MN-anti-miR10b or with the inactive control, MN-scr-miR. No difference in apoptotic rates was observed between experimental and control animals. (b) Tumor cell proliferation in the primary tumors and lymph node metastases. In the presence of miR-10b inhibition, tumor cell proliferation was significantly reduced in the lymph node metastases but not in the primary tumors $(P<0.0026, n=3)$. Data are represented as mean \pm s.d. 